\title{
Research on the Preparation and Performance of Binary Modified Maltodextrin Water-Reducing Agent
}

\author{
Jingzhi Wu, ${ }^{1}$ Min Qiao, ${ }^{2}$ Zhifeng Lyu, ${ }^{2}$ and Qianping Ran ${ }^{1}$ \\ ${ }^{1}$ Jiangsu Research Institute of Building Science, Nanjing 210008, China \\ ${ }^{2}$ State Key Laboratory of High Performance Civil Engineering Materials, Jiangsu Sobute New Materials Co., Ltd., \\ Nanjing 211103, China
}

Correspondence should be addressed to Min Qiao; qiaomin@cnjsjk.cn and Qianping Ran; ranqianping@cnjsjk.cn

Received 20 May 2016; Revised 31 August 2016; Accepted 18 September 2016

Academic Editor: Kestutis Baltakys

Copyright (C) 2016 Jingzhi Wu et al. This is an open access article distributed under the Creative Commons Attribution License, which permits unrestricted use, distribution, and reproduction in any medium, provided the original work is properly cited.

\begin{abstract}
Using maltodextrin (MD) of different dextrose equivalent (DE) values, 1,3-propanesultone, and maleic anhydride as raw materials, a novel binary modified maltodextrin (BMMD) was synthesized and further applied as a water-reducing agent. Its structure was characterized by Fourier transform infrared (FT-IR) and UV. The rheological behavior of the sample solution and strengths for concrete were also determined and the adsorption was tested by TOC. The influence of the process parameters to degree of substitution (DS) and the dosage on the fluidity of cement paste were investigated. The results show that the optimal conditions of sulfonation were the MD of DE $15, m$ (sulfonated agent) $/ m$ (MD) of 1.4, the catalyst amount of $1 \%$ by mass MD, and the reaction time of $12 \mathrm{~h}$; the optimal conditions of esterification were $m$ (esterified agent) $/ m$ (SMD) of 0.6 , the reaction temperature of $90^{\circ} \mathrm{C}$, and the reaction time of $4 \mathrm{~h}$. The optimal dosages of sulfonated maltodextrin (SMD) and BMMD were $0.475 \%$ and $0.45 \%$, respectively. In this dosage, the main dispersion capacity of BMMD attributes to two kinds of anchoring groups $\left(\mathrm{SO}_{3}^{-} \& \mathrm{COO}^{-}\right)$and the appropriate molecular weight of MD.
\end{abstract}

\section{Introduction}

Nowadays, water-reducing agent is an essential admixture for high performance concrete [1-3]. Various types of waterreducing agents have been developed. One of the mostly used types is produced by condensation polymerization, including naphthalene-based superplasticizer, aliphatic plasticizer, and aminosulfonic acid-based plasticizer $[4,5]$. Yet, the incorporating of formaldehyde leads to serious environmental pollution, which is against the sustainable development of our society $[6,7]$. The other type of water-reducing agent is polycarboxylate (PCE) superplasticizer, which exhibits excellent water-reducing ratio and is prepared by environmental friendly reaction - free radical polymerization in pure aqueous media $[8,9]$. However, the key monomers-acrylic acid-and macromonomer containing long polyethylene glycol chain are the products from petrochemical industry, which might be restricted by the limited oil reserves. In this regard, the development for new plasticizers based on natural plants is necessary.
In recent years, some researches focused on the synthesis of plasticizers based on starch that is a renewable and nonpolluting raw material [10]. Various starch esters and ethers have been used in concrete as thickening and waterretention agents. The degree of polymerization of modified starch is about 50-300 [11]. They show a high viscosity at a low concentration in water due to their high molecular weight. Maltodextrin (MD) is a hydrolysis product of starch, whose $\mathrm{DE}$ values are less than 20 . The MDs with different $\mathrm{DE}$ values and different functionalized groups, including sulfonation and carboxymethylation, have been investigated [12-14]. However, the hydroxyl groups of starch have low reactivity; thus it could be hard for the single group modified starch to reach high DS, which limits the dispersing performancein cement paste.

In this study, a dual-groups modified $\mathrm{MD}$ was reported for the first time. BMMD was synthesized by SMD. SMD was the modified MD by sulfonic groups. The influence of the processing parameters on DS and the dosage on the fluidity of cement paste were investigated and were compared with 
The first step:<smiles>CO[C@H]1C(O)[C@H](O)[C@H](O)[C@H]1O</smiles>

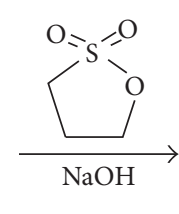<smiles>COC1C(O)C(O)C(O)C1O</smiles>

The second step:

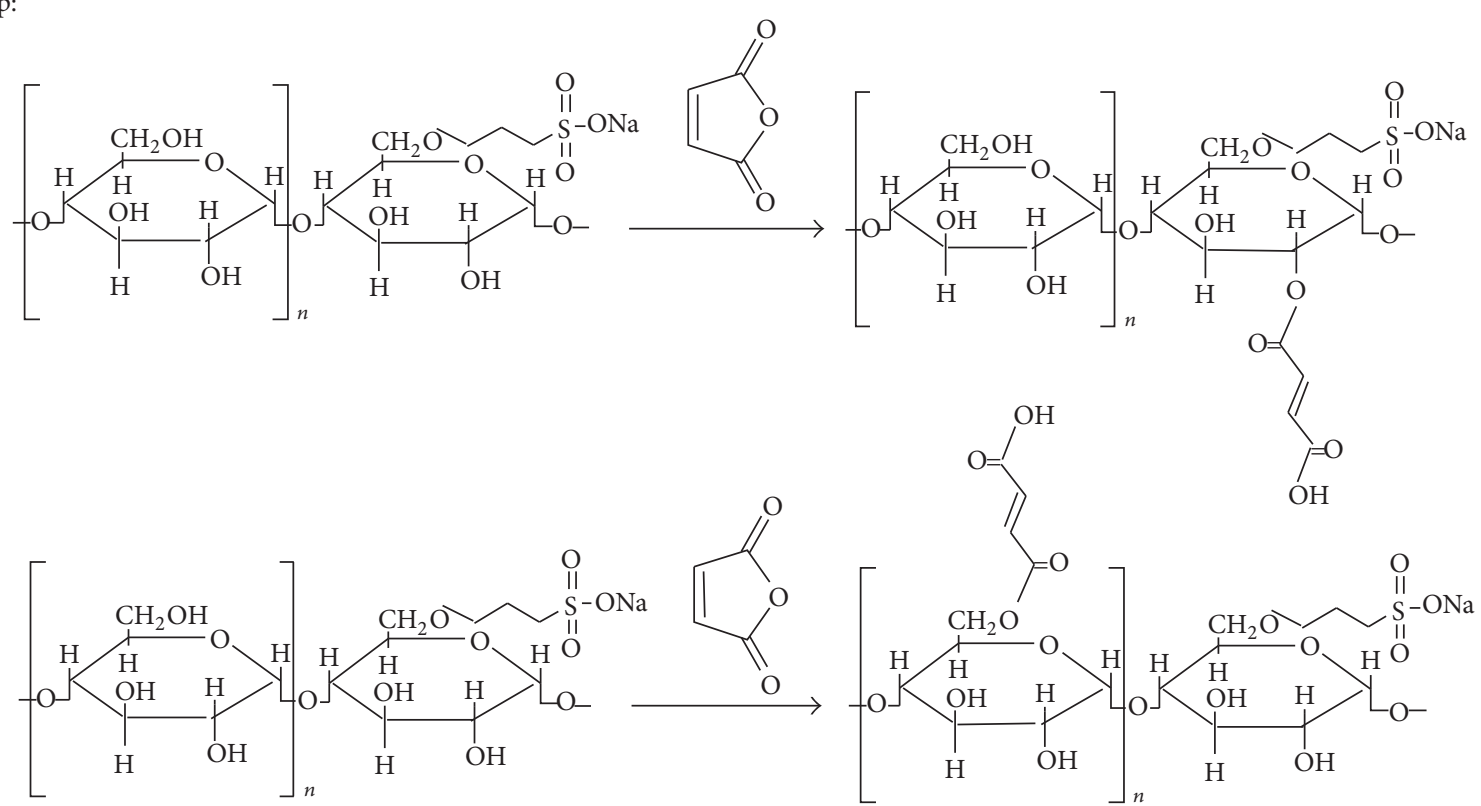

Scheme 1: Preparation process of BMMD.

conventional cement dispersants. FT-IR and UV confirmed the structure of the BMMD, and the rheological behavior of the sample solution and strengths for concrete were also determined. The working mechanism of BMMD compared with SMD was also discussed in detail, which was tested by TOC. The results show that BMMD has higher DS and higher water-reducing ability.

\section{Experimental}

2.1. Materials. An ordinary Portland cement (42.5, Lafarge Cement Co., China) which met the requirements of GB8076 standard was used. Naphthalene superplasticizer (powders) was purchased from Jiangsu Sobute New Materials Co., Ltd. (Jiangsu, China). MDs with different DE values (less than 10\% moisture content) were purchased from Shandong Xiwang Sugar Co., Ltd. (Shandong, China). 1,3-Propanesultone (purity of 99\%) and maleic anhydride (purity of 99\%) were purchased from Aladdin Reagent Co., Ltd. (Shanghai, China). Sodium hydroxide (flake, purity of 96\%) and hydrochloric acid ( $w$ of 36 38\%) were purchased from Chinese Medicine Group Chemical Reagent Co., Ltd. (Shanghai, China). Pyridine (purity of 99.5\%) was purchased from Shanghai Shenbo Chemical Co., Ltd. (Shanghai, China). All the reagents were AR.
2.2. Preparation Methods of BMMD. BMMD was prepared according to the reported method with some modifications [15]. The different $\mathrm{DE}$ values of $\mathrm{MD}$ were dried at $50^{\circ} \mathrm{C}$ in vacuum, added to the pyridine that dissolved in sodium hydroxide and activated for $0.5 \sim 1 \mathrm{~h}$ at room temperature, and added to the sulfonated agent and reacted 10 14 h at room temperature. Then different DS of SMD were obtained after the procedure of washing, filtrating, and drying. SMD and maleic anhydride were mixed and grinded and then heated in water for $4 \mathrm{~h}$ at $90^{\circ} \mathrm{C}$. At last, the BMMD water-reducing agent was obtained and denoted as BMMD. The reaction process might be described by Scheme 1 .

2.3. Test Methods of BMMD. The DS of SMD was determined according to the method of Barium sulfate-gelatin spectrophotometer [16]. The DS of maleic anhydride was determined according to the method of Wurzburg [17].

Fourier Transform Infrared (FT-IR) Spectroscopy. The FTIR spectra of MD, SMD, and BMMD were obtained from samples in $\mathrm{KBr}$ pellets using a Nicolet FT-IR Spectrometer (Impact 410, Madison, WI). The FT-IR spectra were scanned over the wave number range of $2500-500 \mathrm{~cm}^{-1}$. Sample concentration was $0.2 \%$. The carboxyl group content of BMMD was measured by UV spectrophotometry according to the method of Bahk [18]. 
TABLE 1: The optimization process for sulfonation process.

\begin{tabular}{lccccc}
\hline Number & $A$ & $B$ & $C(\%)$ & $D(\mathrm{~h})$ & DS of sulfonation \\
\hline 1 & 10 & 0.7 & 0.8 & 10 & $0.138 \pm 0.005$ \\
2 & 10 & 1.4 & 1.0 & 12 & $0.179 \pm 0.002$ \\
3 & 10 & 2.8 & 1.2 & 14 & $0.161 \pm 0.008$ \\
4 & 15 & 0.7 & 1.0 & 14 & $0.186 \pm 0.006$ \\
5 & 15 & 1.4 & 1.2 & 10 & $0.206 \pm 0.008$ \\
6 & 15 & 2.8 & 0.8 & 12 & $0.175 \pm 0.001$ \\
7 & 20 & 0.7 & 1.2 & 12 & $0.170 \pm 0.003$ \\
8 & 20 & 1.4 & 0.8 & 14 & $0.162 \pm 0.002$ \\
9 & 20 & 2.8 & 1.0 & 10 & $0.175 \pm 0.007$ \\
$K_{1}$ & 0.159 & 0.165 & 0.158 & 0.173 & \\
$K_{2}$ & 0.189 & 0.182 & 0.180 & 0.175 & \\
$K_{3}$ & 0.169 & 0.170 & 0.179 & 0.170 & \\
$R$ & 0.030 & 0.017 & 0.022 & 0.005 & \\
\hline
\end{tabular}

TABLE 2: Effect of the DS of sulfonation on the fluidity of cement paste.

\begin{tabular}{lccccc}
\hline DS of sulfonation & 0.138 & 0.155 & 0.182 & 0.202 & 0.223 \\
\hline Fluidity of cement paste $(\mathrm{mm})$ & 173 & 190 & 205 & 218 & 188 \\
\hline
\end{tabular}

The amount of sample adsorbed was determined by means of a total organic carbon analyzer (TOC), Multi N/C3100 (Analytikjene AG, Germany). That was reference method of Ran [19].

A certain amount of water-reducing agent was added to $87 \mathrm{~g}$ water in advance and then added to the $300 \mathrm{~g}$ cement. The fluidity of cement paste was evaluated by pullout spread of the sample from a flow cone specified according to GB 8077-87. On the basis of the test fluidity of cement paste, $1.5 \mathrm{~g}$ montmorillonite was added to cement. The mortar and concrete performance were measured according to GB 80762008. The maximum diameter of the spread sample and the maximum width perpendicular to that diameter were measured, and the average of these two values was defined as the flow value.

\section{Results and Discussion}

3.1. Effect of the DS of Sulfonation Process. Table 1 shows the optimization process for SMD with $\mathrm{L}_{9}\left(3^{4}\right)$ orthogonal test, and affecting sequence on DS was the MD of DE values $(A)>$ the catalyst amount $(C)>m$ (sulfonated agent) $/ m(\mathrm{MD})(B)$ $>$ reaction time $(D)$. The optimal conditions of sulfonation were the MD of DE $15, m$ (sulfonated agent) $/ m$ (MD) of 1.4, the catalyst amount of $1 \%$ by mass $\mathrm{MD}$, and the reaction time of $12 \mathrm{~h}$.

Table 2 shows the relationship between DS of sulfonation and the fluidity of cement paste. It can be seen that the fluidity of SMD increased with increasing DS when DS $<0.202$. Because $\mathrm{SO}_{3}{ }^{-}$has a strong hydrophilicity that can adsorb on the surface of cement particles, the cement particles were dispersed, the number of $\mathrm{SO}_{3}{ }^{-}$increased, and the adsorption groups of cement particle surface increased; the
TABLE 3: The optimization process for esterification process.

\begin{tabular}{lcccc}
\hline Number & $A$ & $B$ & $C(\%)$ & DS of esterification \\
\hline 1 & 0.4 & 85 & 3 & $0.166 \pm 0.005$ \\
2 & 0.4 & 90 & 4 & $0.208 \pm 0.002$ \\
3 & 0.4 & 95 & 5 & $0.188 \pm 0.005$ \\
4 & 0.6 & 85 & 5 & $0.243 \pm 0.006$ \\
5 & 0.6 & 90 & 3 & $0.285 \pm 0.004$ \\
6 & 0.6 & 95 & 4 & $0.268 \pm 0.007$ \\
7 & 0.8 & 85 & 4 & $0.291 \pm 0.006$ \\
8 & 0.8 & 90 & 5 & $0.324 \pm 0.009$ \\
9 & 0.8 & 95 & 3 & $0.317 \pm 0.008$ \\
$K_{1}$ & 0.187 & 0.233 & 0.253 & \\
$K_{2}$ & 0.265 & 0.272 & 0.256 & \\
$K_{3}$ & 0.311 & 0.258 & 0.255 & \\
$R$ & 0.124 & 0.039 & 0.003 & \\
\hline
\end{tabular}

TABLE 4: Effect of the DS of esterification on the fluidity of cement paste.

\begin{tabular}{lccccc}
\hline DS of esterification & 0.217 & 0.245 & 0.283 & 0.312 & 0.355 \\
\hline Fluidity of cement paste $(\mathrm{mm})$ & 220 & 242 & 265 & 236 & 205 \\
\hline
\end{tabular}

better effect on the water-reducing was therefore obtained. But if the sulfonated agent was excessive, MD would be partially degraded, which is not conducive to water-reducing agent.

3.2. Effect of the DS of Esterification Process. Table 3 shows the optimization process for BMMD with $\mathrm{L}_{9}\left(3^{3}\right)$ orthogonal test, and affecting sequence on DS was $m$ (esterified agent) $/ m$ $(\mathrm{SMD})(A)>$ reaction temperature $(B)>$ reaction time $(C)$. The optimal conditions of esterification were $m$ (esterified agent $) / m(\mathrm{SMD})$ of 0.6 , the reaction temperature of $90^{\circ} \mathrm{C}$, and the reaction time of $4 \mathrm{~h}$.

Table 4 shows the relationship between DS of esterification and the fluidity of cement paste. Take the SMD with the DS of 0.202 as the raw material, different DS of BMMD were prepared. It can be seen that the fluidity of BMMD increased with the increasing DS when DS $<0.283$. Because $\mathrm{COO}^{-}$is a polar group, tailed adsorption was dominant when the number of $\mathrm{COO}^{-}$was low and the fluidity of cement paste was improved. While horizontal adsorption was the main adsorption and DS $>0.283$, the ability of water-reducing agent was lower [20].

3.3. FT-IR. Figure 1 shows that in comparison with IR spectra of MD, SMD, and BMMD, IR spectra of SMD have strong absorption peaks located at $1141 \mathrm{~cm}^{-1}$ and $619 \mathrm{~cm}^{-1}$ both assigned as the characteristic adsorption of $\mathrm{S}=\mathrm{O}$, indicating that sulfonate group was introduced to the MD molecules. Besides the above peaks, BMMD also had another strong absorption peak located at $1734 \mathrm{~cm}^{-1}$, attributed to the characteristic adsorption of $\mathrm{C}=\mathrm{O}$, suggesting that maleic anhydride was introduced to the MD molecule in the acid form. 


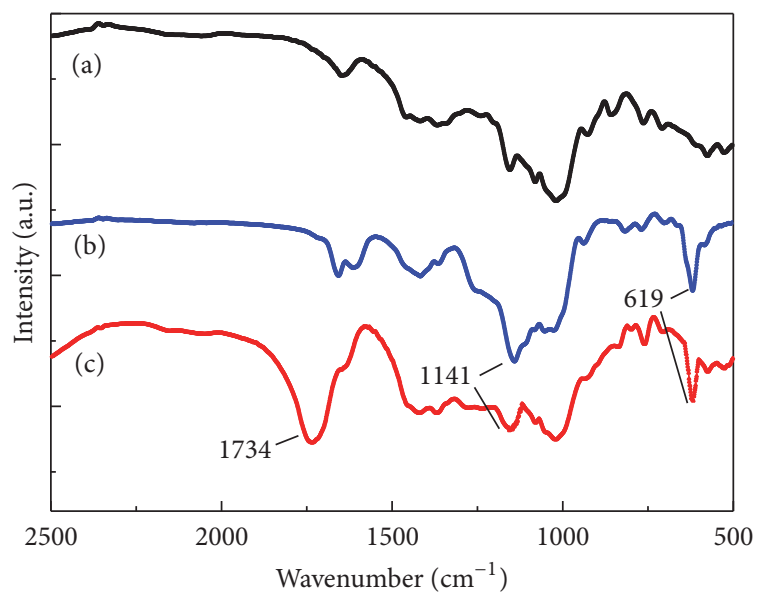

Figure 1: IR spectra of MD (a), SMD (b), and BMMD (c).

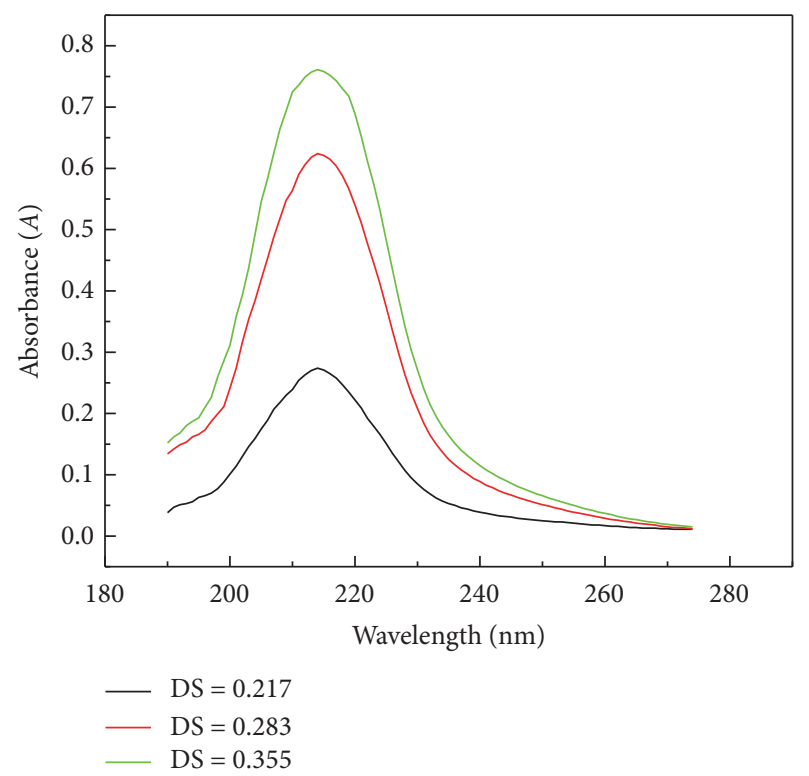

FIgURE 2: UV spectra of different DS of BMMD.

3.4. UV. Figure 2 depicts the difference of UV spectra of BMMD samples with various DS values of $0.217,0.283$, and $0.355(\lambda \max =214 \mathrm{~nm})$. The DS of BMMD increased from 0.217 to 0.355 ; absorbance was also increasing. With increasing DS of esterification, the carboxyl group content was increased.

3.5. Adsorption Behavior. Figure 3 shows adsorption performance of SMD and BMMD. For all the sample, the adsorption curves have the same trend with the adsorbed amount increasing rapidly with the dosage in the range of $0.35 \%-0.6 \%$ of cement mass. Within this period, the surfaces of cement particles are more active and also contain more empty sites. Therefore, the adsorption ratio is relatively high. The adsorption amount of BMMD increases more, because it has two kinds of anchoring groups $\left(\mathrm{SO}_{3}{ }^{-} \& \mathrm{COO}^{-}\right)$.

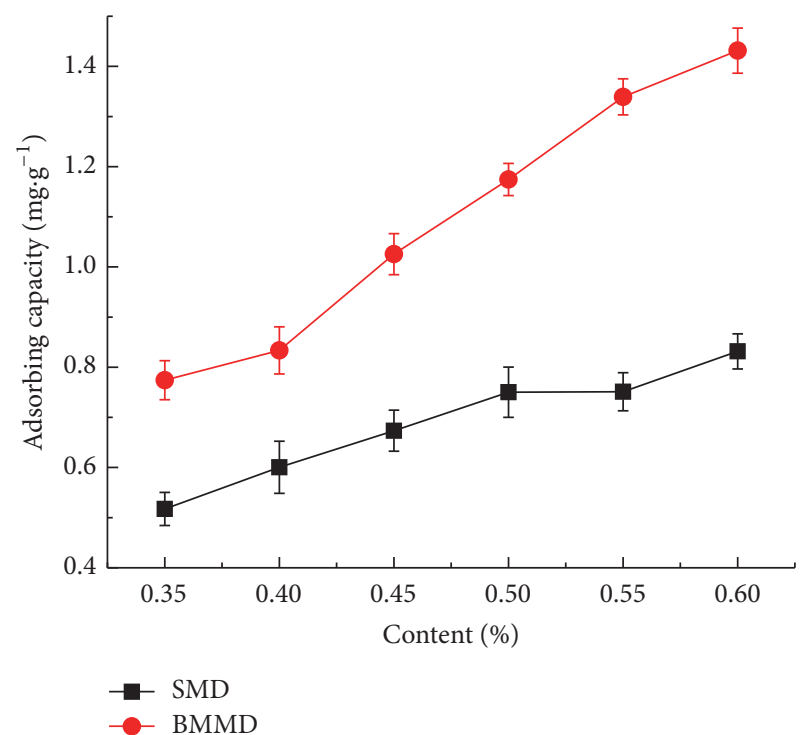

FIGURE 3: Effect of SMD and BMMD content on the adsorption capacity.

TABLE 5: Setting time.

\begin{tabular}{lcc}
\hline & Blank & BMMD \\
\hline Time of setting/h & & \\
Initial set & 2.96 & 11.35 \\
Final set & 3.88 & 15.28 \\
\hline
\end{tabular}

3.6. Setting Time/Concrete Performance. Under the same water requirement for normal consistency of cement paste, setting time was measured on BMMD and white cement paste with content was $0.45 \%$ of cement mass. The results were shown in Table 5; BMMD has a strong retarding effect on cement paste. Concrete performance of naphthalene series and BMMD was compared; the results were shown in Table 6; the water-reducing and slump retention capability of BMMD are obviously better than those of naphthalene series. In terms of strength, because its strong retarding effect, early strength is slightly lower than naphthalene series, but it has no effect on the ultimate strength.

3.7. Anti-Clay Performance. Figure 4 shows anticlay performance of BMMD and naphthalene series. In the first set of experiments, the dosage of water-reducing agent was $0.5 \%$ of cement mass. In a second set of experiments, $1.5 \mathrm{~g}$ montmorillonite was added to cement extra. After adding montmorillonite, the fluidity of cement paste of naphthalene series was reduced significantly, and the fluidity of cement paste of BMMD was essentially unchanged. Montmorillonite has a large layer spacing and naphthalene series is straightchain structure, so it is easy to enter layer spacing thereby reducing the water-reducing effect. BMMD is limb structure, as the role of the branched dextrin; its volume increases several times to several times; therefore, it would not enter layer spacing. 
TABLE 6: Concrete performance.

\begin{tabular}{|c|c|c|c|c|c|c|c|}
\hline \multirow{2}{*}{ water-reducing agent } & \multirow{2}{*}{ Content (\%) } & \multirow{2}{*}{ Gas content (\%) } & \multicolumn{2}{|c|}{ Slump constant/divergence $(\mathrm{cm})$} & \multicolumn{3}{|c|}{ Pressive strength $(\mathrm{MPa})$} \\
\hline & & & Initial & $50 \mathrm{~min}$ & $3 \mathrm{~d}$ & $7 \mathrm{~d}$ & $28 \mathrm{~d}$ \\
\hline Naphthalene series & 0.45 & 2.8 & $23.7 / 48$ & 15.9 & 34.8 & 50.2 & 70.8 \\
\hline BMMD & 0.45 & 3.0 & $24.1 / 56$ & $19.8 / 35$ & 31.2 & 50.2 & 72.1 \\
\hline
\end{tabular}

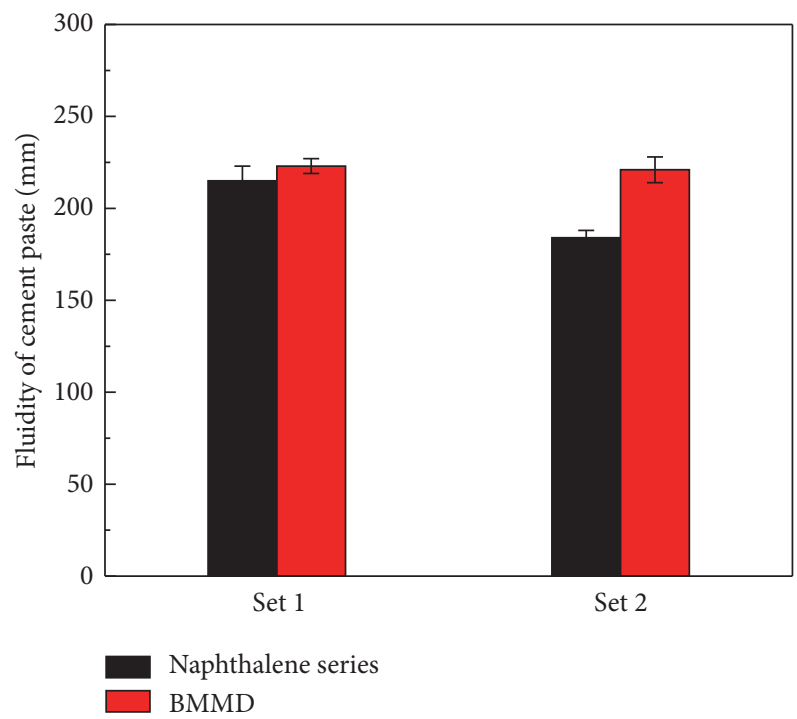

FIGURE 4: Anticlay performance of BMMD and naphthalene series.

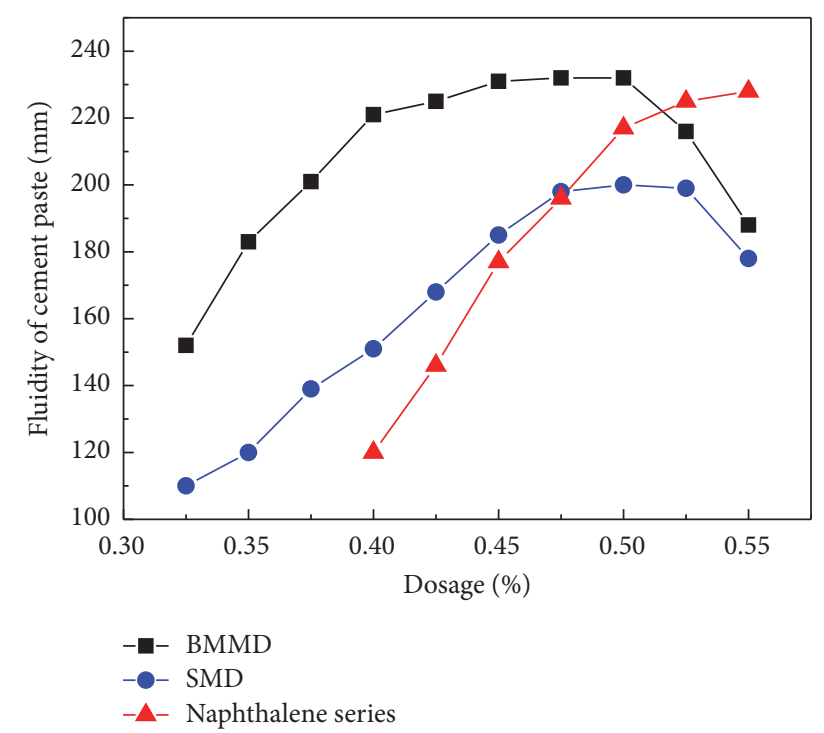

FIGURE 5: Effect of the water-reducing agent added on the fluidity of cement paste.

3.8. Effect of Dosage of Water-Reducing Agent on the Fluidity of Cement Paste. Figure 5 shows that the water-reducing agent with the same variety of dosage can significantly improve the fluidity of cement paste. Fixing the water-binder ratio as 0.29 , the fluidity of cement paste with SMD increased from 110 to
$200 \mathrm{~mm}$ while the dosage increased from $0.325 \%$ to $0.55 \%$ and BMMD increased from 152 to $232 \mathrm{~mm}$. Naphthalene series water-reducing agent increased from 120 to $228 \mathrm{~mm}$ when the dosage increased from $0.4 \%$ to $0.55 \%$. But the fluidity of cement paste could not increase with increasing dosage of SMD and BMMD. On the contrary, it descended when the dosages were more than $0.525 \%$ and $0.5 \%$, respectively. This phenomenon can be explained by the significant role of viscosity as the dosage increased. Cement paste would bleed when the dosage of naphthalene series was higher than $0.55 \%$. Thus, the optimal dosages of SMD and BMMD were $0.475 \%$ and $0.45 \%$, respectively. In this dosage, the effect of waterreducing was better than that of commonly used naphthalene series.

\section{Conclusions}

(1) SMD and BMMD were synthesized, and BMMD was proved to be a novel kind of water-reducing agent with high efficiency of water-reducing performance.

(2) The dispersing capacity of SMD and BMMD is in association with DS. The optimal dosages of SMD and BMMD were $0.475 \%$ and $0.45 \%$, respectively. In these dosages, the effect of water-reducing agent was better than that of naphthalene series.

(3) The dispersing capacity of BMMD on the fluidity of cement paste may attribute to the electrostatic repulsion rather than steric hindrance repulsion. Therefore, the electrostatic repulsion of BMMD is stronger due to two kinds of anchoring groups, which results in better dispersing capacity for BMMD compared with SMD. But also, the appropriate molecular weight of $\mathrm{MD}$ is needed, which provides a certain steric hindrance repulsion. BMMD has good resistance of clay.

\section{Competing Interests}

The authors declare that there is no conflict of interests regarding the publication of this article.

\section{Acknowledgments}

This study is financially supported by the National Natural Science Foundation of China (nos. 51302114 and 51408271) and the Natural Science Foundation of Jiangsu Province of China (no. BK20131010). 


\section{References}

[1] J. Plank, C. Schroefl, M. Gruber, M. Lesti, and R. Sieber, "Effectiveness of polycarboxylate superplasticizers in ultra-high strength concrete: the importance of PCE compatibility with silica fume," Journal of Advanced Concrete Technology, vol. 7, no. 1, pp. 5-12, 2009.

[2] M. Collepardi, "Admixtures used to enhance placing characteristics of concrete," Cement and Concrete Composites, vol. 20, no. 2-3, pp. 103-112, 1998.

[3] R. Parichatprecha and P. Nimityongskul, "Analysis of durability of high performance concrete using artificial neural networks," Construction and Building Materials, vol. 23, no. 2, pp. 910-917, 2009.

[4] L. Huynh, D. A. Beattie, D. Fornasiero, and J. Ralston, "Effect of polyphosphate and naphthalene sulfonate formaldehyde condensate on the rheological properties of dewatered tailings and cemented paste backfill," Minerals Engineering, vol. 19, no. 1, pp. 28-36, 2006.

[5] E. Sakai, K. Yamada, and A. Ohta, "Molecular structure and dispersion-adsorption mechanisms of comb-type superplasticizers used in Japan," Journal of Advanced Concrete Technology, vol. 1, no. 1, pp. 16-25, 2003.

[6] R. Loos, J. Riu, M. C. Alonso, and D. Barceló, "Analysis of polar hydrophilic aromatic sulfonates in waste water treatment plants by CE/MS and LC/MS," Journal of Mass Spectrometry, vol. 35, no. 10, pp. 1197-1206, 2000.

[7] R. Loos, M. C. Alonso, and D. Barceló, "Solid-phase extraction of polar hydrophilic aromatic sulfonates followed by capillary zone electrophoresis-UV absorbance detection and ion-pair liquid chromatography-diode array UV detection and electrospray mass spectrometry," Journal of Chromatography A, vol. 890, no. 2, pp. 225-237, 2000.

[8] Z.-N. Xu, G.-M. Xiao, and J.-N. Zhou, "Kinetics of carboxylic acid terpolymer high performance water-reducing agent," Polymeric Materials Science and Engineering, vol. 26, no. 1, pp. 26-32, 2010.

[9] D. Zhiqin, C. Guoxin, Z. Yeran et al., "Study on preparation and properties of polycarboxylate superplasticizer with earlyage strength," Concrete, vol. 259, no. 5, pp. 94-96, 2011.

[10] J. Loksuwan, "Characteristics of microencapsulated $\beta$-carotene formed by spray drying with modified tapioca starch, native tapioca starch and maltodextrin," Food Hydrocolloids, vol. 21, no. 5-6, pp. 928-935, 2007.

[11] M. C. Vieira, D. Klemm, L. Einfeldt, and G. Albrecht, "Dispersing agents for cement based on modified polysaccharides," Cement and Concrete Research, vol. 35, no. 5, pp. 883-890, 2005.

[12] F. Tegiacchi and B. Casu, "Alkylsulfonierte polysaccharide und diese enthaltende moertel-und betonmischungen," DE3406745A1, 1984.

[13] D.-F. Zhang, B.-Z. Ju, S.-F. Zhang, and J.-Z. Yang, "The study on the synthesis and action mechanism of starch succinate half ester as water-reducing agent with super retarding performance," Carbohydrate Polymers, vol. 71, no. 1, pp. 80-84, 2008.

[14] L. Einfeldt, G. Albrecht, A. Kem et al., "Use of water-soluble polysaccharide derivatives as dispersing agents for mineral binder suspensions," US 20040103824AI, 2004.

[15] C. Fa, H. Guili, Y. Changchun et al., "Synthesis and properties of sulfonated starch as superplasticizer," Fine Chemicals, vol. 23, no. 7, pp. 711-716, 2006.

[16] M. Yamamoto, E. Takahashi, H. Tamura et al., "Antitumor activity of edible marine algae: effect of crude fucoidan fractions prepared from edible brown seaweeds against L-1210 leukemia ," Eleventh International Seaweed Symposium, vol. 22, pp. 145-148, 1962.

[17] O. B. Wurzburg, "Starch derivatives and modification," in Methods in Carbohydrate Chemistry, pp. 286-288, Academic Press, New York, NY, USA, 4th edition, 1964.

[18] Y. K. Bahk, X. He, E. Gitsis, Y.-Y. Kuo, N. Kim, and J. Wang, "Enhanced dispersion stability and mobility of carboxylfunctionalized carbon nanotubes in aqueous solutions through strong hydrogen bonds," Journal of Nanoparticle Research, vol. 17, no. 10, article 396, 2015.

[19] Q. Ran, P. Somasundaran, C. Miao, J. Liu, S. Wu, and J. Shen, "Effect of the length of the side chains of comb-like copolymer dispersants on dispersion and rheological properties of concentrated cement suspensions," Journal of Colloid and Interface Science, vol. 336, no. 2, pp. 624-633, 2009.

[20] P. Zhao, Y. Yan, Z.-H. Hu, and T.-T. Wang, "Synthesis on oxidized-acidified-etherified starch as superplasticizer," Journal of Wuhan University of Technology, vol. 33, no. 11, pp. 33-38, 2011. 

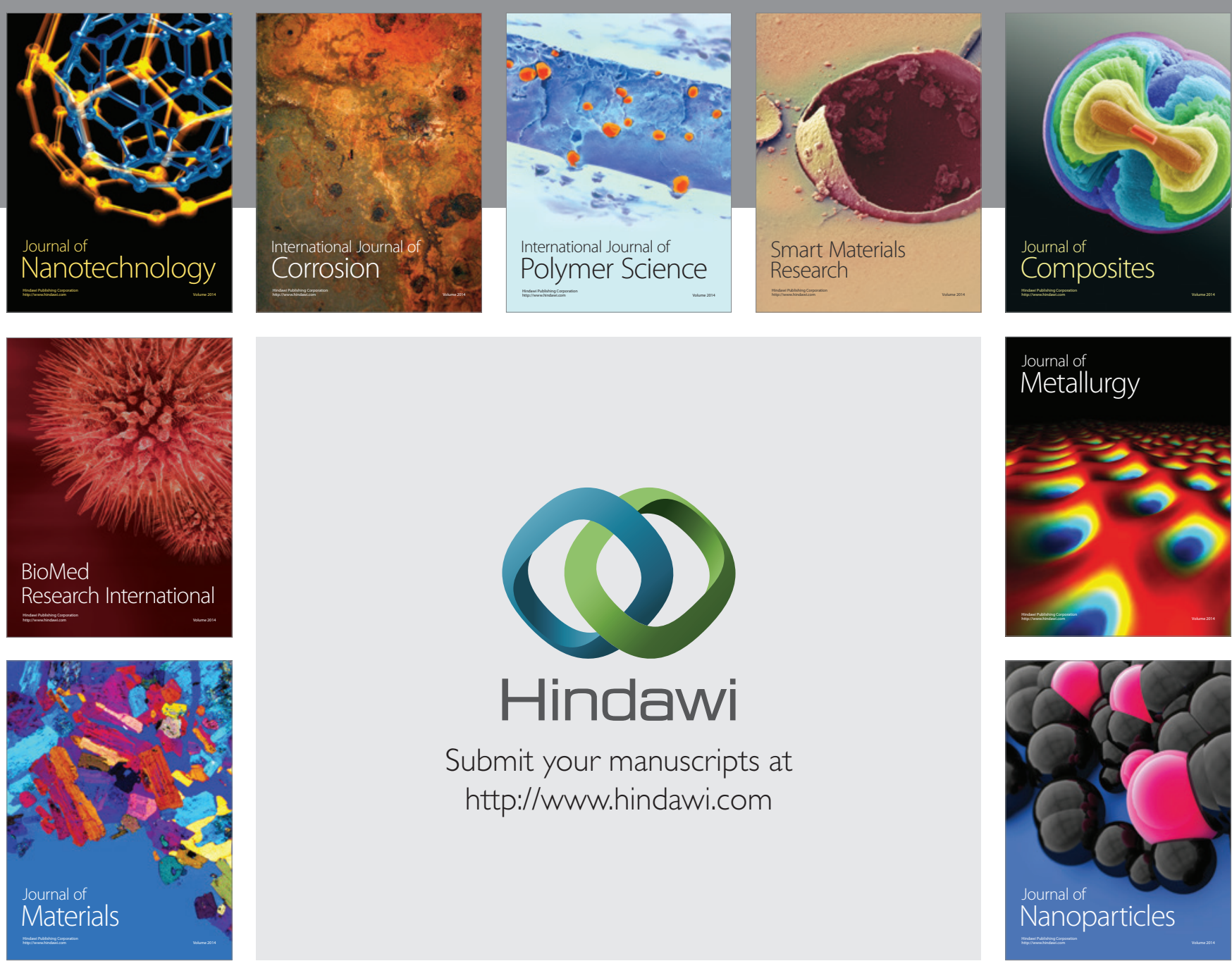

\section{Hindawi}

Submit your manuscripts at

http://www.hindawi.com

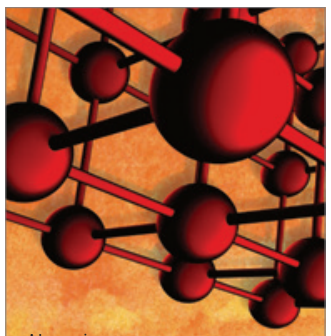

Materials Science and Engineering
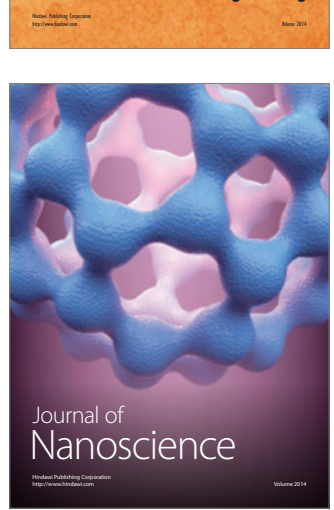
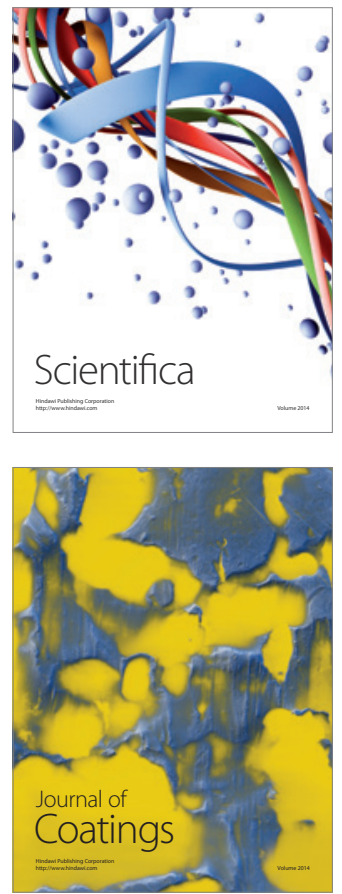
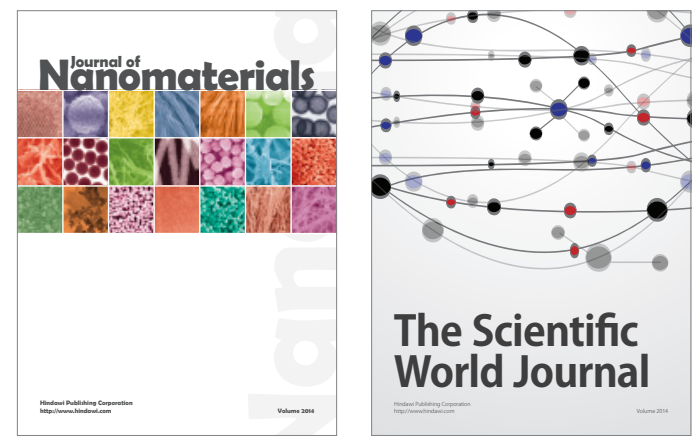

The Scientific World Journal
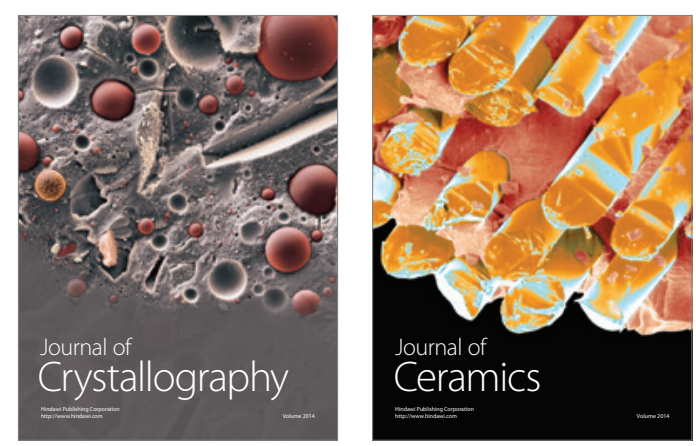
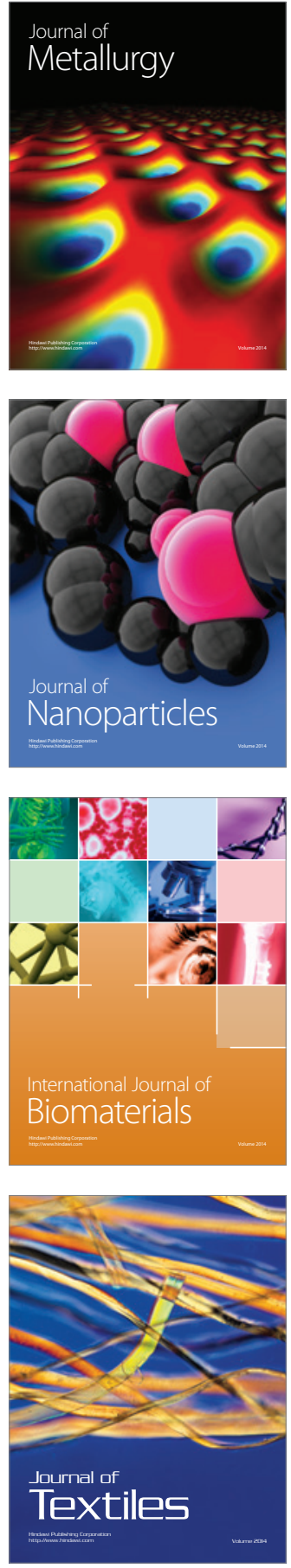